\title{
The impact of FADS genotype on fatty acid status in older adults
}

\author{
C. O’Neill ${ }^{1}$, A. Jennings ${ }^{1}$, N. Tejera Hernandez ${ }^{1}$, R. Gillings ${ }^{1}$, J. Sinnwell ${ }^{2}$, S. Fairweather-Tait ${ }^{1}$, \\ A. Cassidy ${ }^{1}$ and AM Minihane ${ }^{1}$ \\ ${ }^{1}$ Nutrition Department, University of East Anglia, Norwich NR4 7TJ, UK and ${ }^{2}$ Department of Health Sciences \\ Research, Mayo Clinic, Minnesota, USA
}

This abstract was awarded an oral presentation prize sponsored by Sugar Nutrition UK and the Food Safety Authority of Ireland.

The objective of this research is to establish the impact of fatty acid desaturase (FADS) gene variants on fatty acid status at baseline in the NU-AGE cohort. The NU-AGE project (EU FP7) involves 1250 older adults (aged 65-79 years) and aims to examine the impact of a year-long whole diet intervention (including advice on intakes of the n-3 fatty acids, eicosapentaenoic acid (EPA) and docosahexaenoic acid (DHA)) on chronic low grade inflammation and overall cardiovascular health [1]. The FADS enzyme is responsible for the desaturation of fatty acids and the bioconversion of essential fatty acids to long chain-polyunsaturated fatty acids, including EPA and DHA. A total of $\mathrm{n}=140$ participants are included in the current analysis. 10 tagging SNPs were selected, using HapMap, and include rs1535, rs174589, rs2524299, rs174605, rs174616, rs174570, rs526126, rs968567, rs174602 and rs498793. Haplotypes and subhaplotypes were also statistically reconstructed from these 10 SNPs, using Haplostats. Participants with the homozygous minor genotype for several of the FADS SNPs had significantly $(\mathrm{p}<0.05)$ higher plasma linoleic acid (LA) and significantly lower arachidonic acid (AA), EPA and DHA status, as well as significantly lower desaturase activity (measured by a product-to-precursor ratio of AA/ LA) compared to participants with either the homozygous major genotype or the heterozygous genotype (Figure 1 gives impact of rs174605 on EPA and DHA as an example).

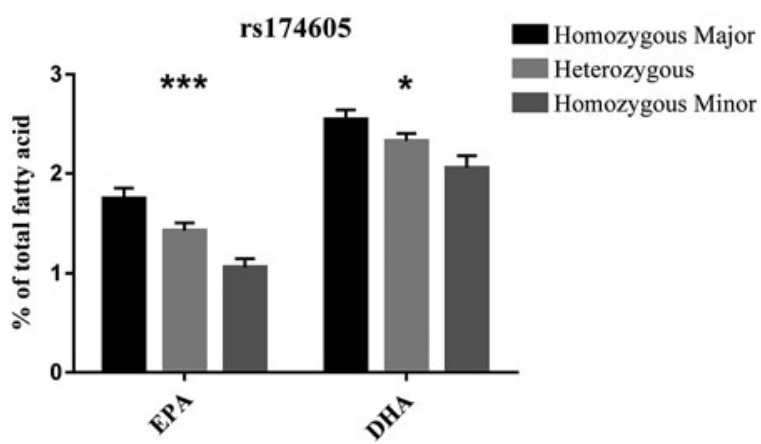

Figure 1. The effects of FADS SNP rs174605 on EPA and DHA plasma status in older adults.

Furthermore, the most common haplotype (containing mostly major alleles and occurring in $26 \cdot 6 \%$ of the cohort) was associated with significantly lower LA plasma levels (up to $9 \%$ increase) and significantly higher EPA (up to $38 \%$ ) and DHA (up to $14 \%$ ) status compared to haplotypes with a higher frequency of minor alleles.

In conclusion, common FADS genotypes may be significant determinants of habitual EPA and DHA status in older adults. However, further research is required in larger sample sizes to determine such effects, as well as to examine the impact of the FADS genotype on tissue function and overall health.

1. Berendsen A., et al., A parallel randomized trial on the effect of a healthful diet on inflammageing and its consequences in European elderly people: design of the NU-AGE dietary intervention study. Mech Ageing Dev, 2013. 